\section{Ruptured abdominal aortic aneurysm presented as Cullen's sign and Grey Turner's sign}

A 60-year-old male with history of hypertension and atrial flutter was sent to our emergency department due to right flank pain and cold sweating. His conscious was clear without fever or abdominal pain. Hypotension (blood pressure: $73 / 52 \mathrm{~mm} \mathrm{Hg}$ ) and tachycardia (heart rate: 142 beats per minute) were detected at triage with marked cold sweating. Bruising around umbilicus (Cullen's sign) and flanks (Grey Turner's sign) were noted (Fig. 1). Bedside sonography showed dilated abdominal aorta $(6 \mathrm{~cm})$ with intramural hematoma and abdominal computed tomography angiography indicated AAA ruptured into vena cava with fistula formation and hemoretroperitoneum (Fig. 2). The patient received fluid resuscitation to maintain systolic blood pressure ranging from 80 to $100 \mathrm{~mm} \mathrm{Hg}$ for hypotensive hemostasis immediately (1). Emergency blood transfusion without cross-matching with 0 -type packed red blood cells and airway protection with endotracheal intubation and ventilator support were all accomplished in a timely manner. The cardiovascular surgeon performed endovascular aneurysm repair (EVAR) for him 68 min later after arriving triage, and then, he was admitted to intensive care unit and successfully discharged 50 days after operation with mild weakness and numbness of the lower limbs.

Rupture of AAA is life-threatening even with prompt treatment. Even with typical symptoms and signs, ruptured AAA may be misdiagnosed as renal colic, perforated viscus, diverticulitis,

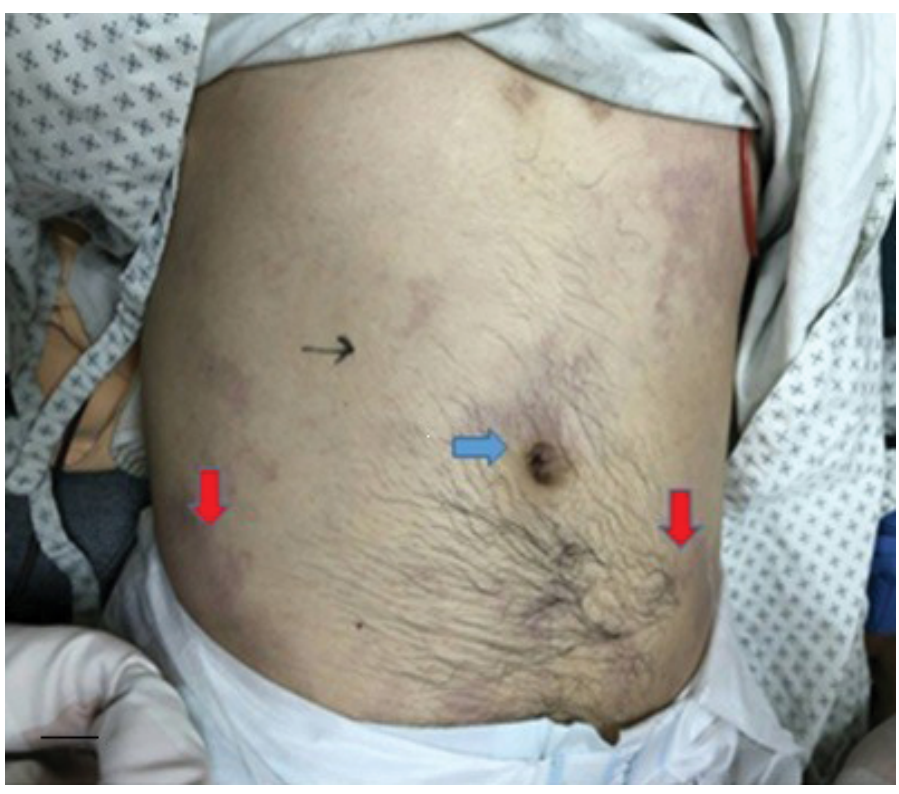

Figure 1. Bruising around the umbilicus (Cullen's sign, blue arrow) and the flanks (Grey Turner's sign, red arrow)
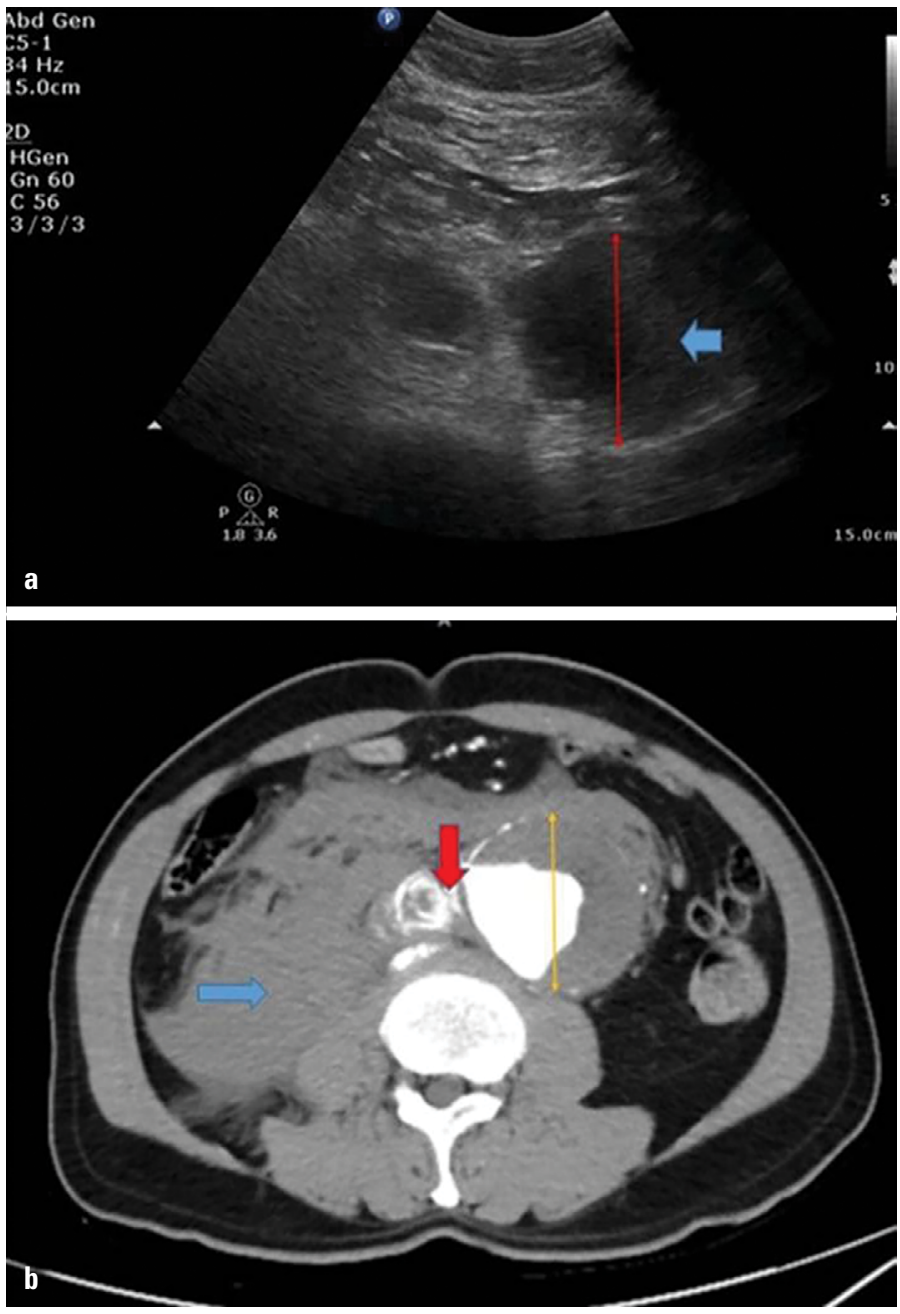

Figure 2. Dilated abdominal aorta $(6 \mathrm{~cm}$, red double arrow) with intramural hematoma (Short blue arrow) and abdominal aortic aneurysm (Orange double arrow) rupture into vena cava with fistula formation (Red arrow) and hemoretroperitoneum (Long blue arrow)

gastrointestinal hemorrhage, or ischemia bowel disease with a ratio of $30 \%$. Patients with risk factors, such as male sex, age between 65 and 80 years, Caucasian ethnicity, tobacco use, and atherosclerosis should draw our attention to this diagnosis (2).

Informed consent: Written informed consent was obtained from the patient for the publication of the case report and the accompanying images.

\section{References}

1. Hamilton $\mathrm{H}$, Constantinou J, Ivancev $\mathrm{K}$. The role of permissive hypotension in the management of ruptured abdominal aortic aneurysms. J Cardiovasc Surg (Torino) 2014; 55: 151-9.

2. Singh K, Bønaa KH, Jacobsen BK, Bjørk L, Solberg S. Prevalence of and risk factors for abdominal aortic aneurysms in a populationbased study : The Tromsø Study. Am J Epidemiol 2001; 154: 236-44. 
(iD) Che-Yu Su, (D) Chi-Wei Lee', (D) Chun-Yen Huang Department of Emergency Medicine, Kaohsiung Medical University Hospital, Kaohsiung Medical University; Kaohsiung-Taiwan

'Institute of Medical Science and Technology, National Sun Yat-Sen University; Kaohsiung-Taiwan

Address for Correspondence: Che-Yu Su, MD,

Department of Emergency Medicine,

Kaohsiung Medical University Hospital,

Kaohsiung Medical University;

No.100,

Tzyou 1st Road Kaohsiung 807,

Kaohsiung-Taiwan

Phone: 886-7-3121101 ext: 7553

E-mail:money0967@gmail.com

(C) Copyright 2020 by Turkish Society of Cardiology - Available online

at www.anatolicardiol.com

DOI:10.14744/AnatolJCardiol.2019.11786

\section{Percutaneous treatment of the Gerbode defect causing heart failure after mitral}

\section{valve surgery}

A 45-year-old female who underwent mechanical mitral valve (MV) replacement 10 years before presentation was hospitalized for decompensated heart failure. Transthoracic echo-
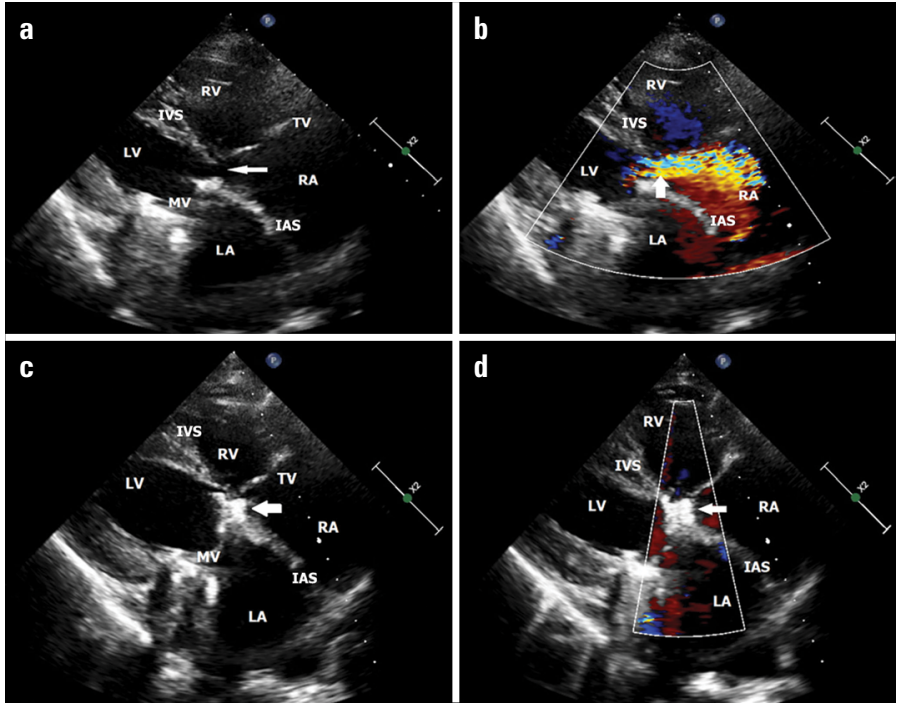

Figure 1. (a and b) Transthoracic echocardiographic images showing the Gerbode defect (GD) in the region marked with an arrow. (c) Image of the Amplatzer Duct Okluder-1 (ADO-1) in the postoperative GD region. (d) AD0-1 preventing the GD-related passage

cardiography (TTE) revealed normal left ventricular diameters and ejection fraction, dilated left atrium, dilated right atrium (RA), and dilated right ventricle. Moderate tricuspid valve (TV) regurgitation was observed, and pulmonary artery systolic pressure was $65 \mathrm{mmHg}$. MV functions were within normal limits. However, a defect between left ventricle (LV) and RA, which
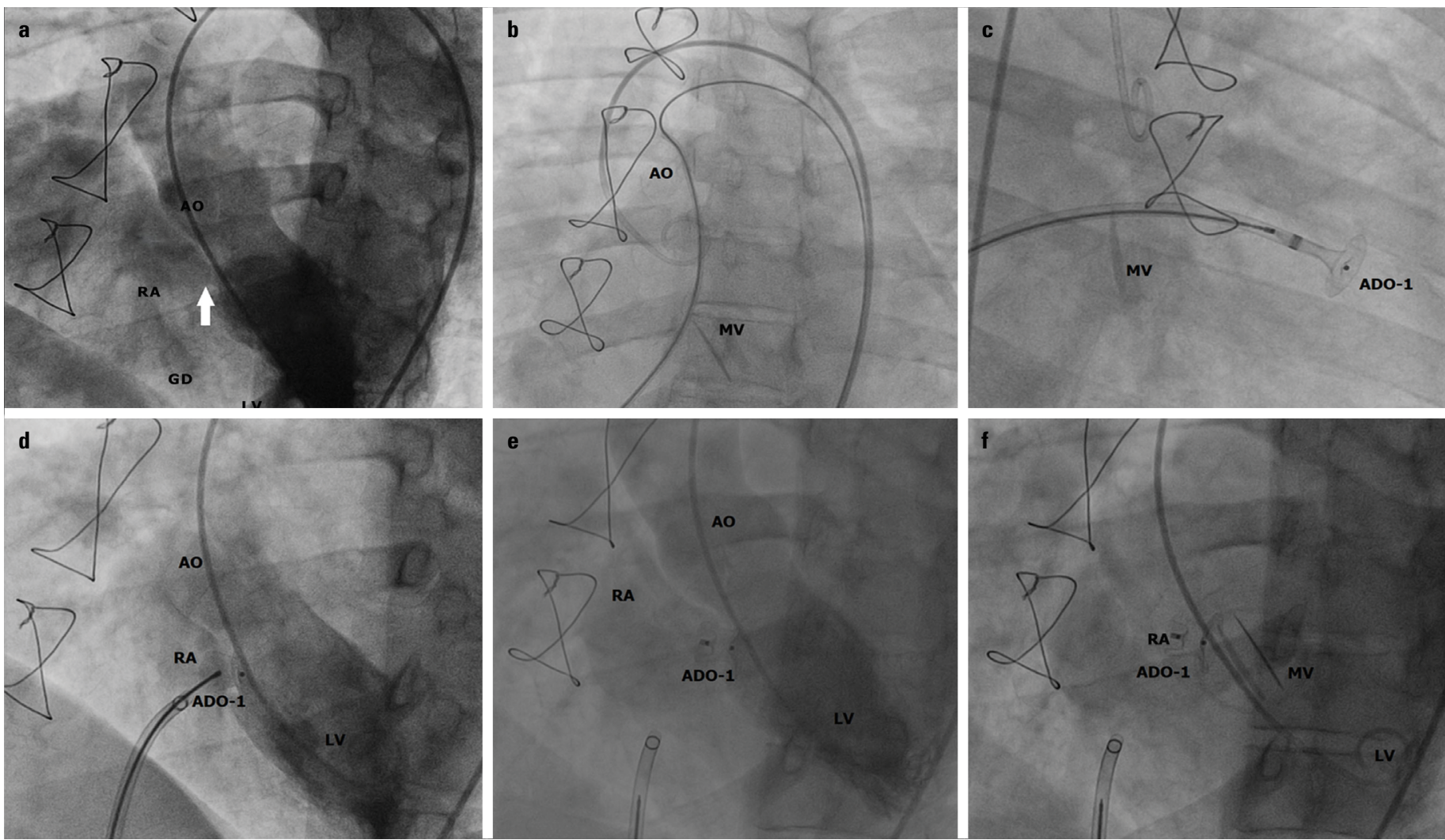

Figure 2. (a) Arrow indicates a connection between left ventricle and right atrium via the Gerbode defect. (b-d) Images of the Amplatzer Duct Okluder-1 (AD0-1) placement procedure. (e) Left ventriculography after releasing AD0-1. (f) Association of the mitral valve and AD0-1 